\title{
Preoperative maximum standardized uptake value and carbohydrate antigen 19- 9 were independent predictors of pathological stages and overall survival in Chinese patients with pancreatic duct adenocarcinoma
}

Xinjin $\mathrm{Gu}^{1 \dagger}$, Ruiquan Zhou ${ }^{2 \dagger}$, Chenggang $\mathrm{Li}^{1 \dagger}$, Rong Liu ${ }^{1,2^{*}}$, Zhiming Zhao ${ }^{1}$, Yuanxing Gao ${ }^{1}$ and Yong Xu ${ }^{1}$

\begin{abstract}
Background: Purpose of this study was to analyze whether preoperative maximum standardized uptake value (SUVmax) and carbohydrate antigen 19-9 (CA19-9) levels might provide prognostic information in Chinese patients with pancreatic duct adenocarcinoma (PDAC) after pancreaticoduodenectomy (PD).

Methods: Standard PD was performed on 109 patients with PDAC by the same operative team, and all patients received preoperative positron emission tomography/computed tomography examination and blood test.

Results: Patients had a mean age of $59 \pm 9.35$ years. Females accounted for 38.5\%. Mean levels of SUVmax, carcinoembryonic antigen (CEA) and CA19-9 were $5.70 \pm 2.76,3.95 \pm 4.16 \mathrm{ng} / \mathrm{mL}$ and $321.62 \pm 780.71 \mathrm{kU} / \mathrm{L}$. In univariate Logistic regression analysis, preoperative SUVmax, CEA and CA19-9 levels ( $p<0.05$ for all) rather than other preoperative variables ( $p>0.05$ for all) were significantly related to AJCC stages. Multivariate Logistic regression analysis showed that preoperative SUVmax and CA19-9 levels ( $p<0.05$ for all) rather than other preoperative variables $(p>0.05$ for all) were significantly associated with AJCC stages. Mean overall survival (OS) was $21 \pm 14.50$ months. In univariate Cox regression analysis, age, SUVmax, CEA and CA19-9 levels before operation ( $p<0.05$ for all) rather than other preoperative variables ( $p>0.05$ for all) were significantly related to OS. Multivariate Cox regression analysis showed that age, SUVmax and CA19-9 levels before operation $(p<0.05$ for all) rather than other preoperative variables ( $p>0.05$ for all) were significantly associated with OS.
\end{abstract}

Conclusions: This study demonstrated that preoperative SUVmax and CA19-9 levels independently predicted pathological stages and OS of patients with PDAC after PD. These preoperative variables might have significant prognostic implication in patients with PDAC after PD. Patients with abnormal SUVmax and CA19-9 levels should be paid special attention to in operative strategy and perioperative management.

Keywords: Carbohydrate antigen 19-9, Maximum standardized uptake value, Pathological stages, Overall survival, Pancreatic duct adenocarcinoma, Pancreaticoduodenectomy

\footnotetext{
* Correspondence: liurong301@126.com

${ }^{+}$Xinjin Gu, Ruiquan Zhou and Chenggang Li contributed equally to this

work.

${ }^{1}$ Department of Hepatobiliary and Pancreatic Surgical Oncology, Chinese

People's Liberation Army General Hospital, Beijing 100853, China

${ }^{2}$ School of Medicine, Nankai University, Tianjin 300071, China
}

(c) The Author(s). 2019 Open Access This article is distributed under the terms of the Creative Commons Attribution 4.0 International License (http://creativecommons.org/licenses/by/4.0/), which permits unrestricted use, distribution, and

reproduction in any medium, provided you give appropriate credit to the original author(s) and the source, provide a link to the Creative Commons license, and indicate if changes were made. The Creative Commons Public Domain Dedication waiver (http://creativecommons.org/publicdomain/zero/1.0/) applies to the data made available in this article, unless otherwise stated. 


\section{Background}

Pancreatic duct adenocarcinoma (PDAC) has not only an increasing incidence in many countries, but also the worst prognosis in digestive tract malignancies [1]. In the United States, it is the fourth most common cause of cancer-related mortality and has a 5-year survival rate of less than 5\% [1]. As shown in Cancer Statistics in China (2015), its new cases were estimated to be 90.1 thousands and new deaths were estimated to be 79.4 thousands in China. Pancreaticoduodenectomy (PD) is an effective treatment for patients with PDAC and achieves a 5-year survival rate of approximately 15 to $40 \%$ [2]. However, regardless of clinically unresectable PDAC (85-90\%), these patients with PDAC still have poor prognosis after PD, with a median survival of 11-19 months [3-5]. Early and individualized operative strategy and perioperative management have reduced perioperative mortality of patients with PDAC after $\mathrm{PD}$ to $<5 \%[6,7]$. Preoperative variables would be of great significance to stratify the patients with PDAC and predict the prognosis of patients after $\mathrm{PD}$, and thus promote the early and individualized operative strategy and perioperative management [8].

The most widely applied ${ }^{18}$ F-fluorodeoxyglucose $\left({ }^{18} \mathrm{~F}-\mathrm{FDG}\right)$ positron emission tomography/computed tomography (PET/CT)-derived variable designed to measure tracer accumulation is the maximum standardized uptake value (SUVmax), which quantifies the rate of glucose metabolic uptake in tumor cells [9]. Recent studies have considered the SUVmax to be beneficial as prognostic factors in patients with PDAC [10]. However, there are still limited studies regarding the prognostic value of SUVmax in Chinese patients with PDAC after PD. Meanwhile, carbohydrate antigen 19-9 (CA19-9), a sialyated Lewis blood group antigen, is expressed in pancreatic ductal cells [11]. Preoperative CA19-9 levels have been shown to be associated with a significant improvement in postoperative survival in patients with PDAC [12]. However, only a small number of studies have investigated the potential role of preoperative CA19-9 levels as a prognostic variable in Chinese patients with PDAC after PD [13]. Purpose of the current study was to analyze whether preoperative SUVmax and CA19-9 levels might provide meaningful prognostic information in Chinese patients with PDAC after PD.

\section{Methods}

\section{Study patients}

Between May 2010 and May 2016, 121 patients were identified from a prospectively maintained database at Department of Hepatobiliary and Pancreatic Surgical Oncology, Chinese People's Liberation Army General Hospital, and enrolled into the current study. Inclusion criteria: 1) with pancreatic head cancer; 2) with PDAC confirmed by pathological results; 3) with operable PDAC; and 4) with open PD. There were 12 patients lost during the follow-up and excluded in the current study. Finally, the current study included 109 patients.

\section{Before operation}

Patient demographics, histories and symptoms were obtained before operation. All participants were injected with ${ }^{18}$ F-FDG $(5.55 \mathrm{MBq} / \mathrm{kg})$ and scanned by Siemens Biograph 64 high definition PET/CT. Scanned area ranged from the skull base to the upper femur, and obtained image was reconstructed by Ordered Subset Expectation Maximization. Preoperative SUVmax levels were measured from the region of interest in ${ }^{18} \mathrm{~F}$-FDG PET/CT image through semi-quantity analysis. Venous blood samples were drawn from all participants, and serum hemoglobin, albumin, total bilirubin, carcino-embryonic antigen (CEA) and CA19-9 were tested using automatic electrochemical luminescence immunoassay method. No patient received chemotherapy or radiotherapy before operation.

\section{Operative process}

All patients underwent standard PD performed by the same operative team at Department of Hepatobiliary and Pancreatic Surgical Oncology, Chinese People's Liberation Army General Hospital. Supporting tubes were routinely placing in the pancreatic duct for external drainage and removed within one week. The range of lymph node dissection included group 5, 8, 12, 13, 14, and 17 lymph nodes. When the tumor invaded the superior mesenteric vein, partial resection and reconstruction of the superior mesenteric vein were selectively performed according to the intraoperative conditions. However, none of the patients included in the current study had resection and reconstruction of the superior mesenteric artery.

\section{After operation}

All specimens were fixed in the formalin for 24-48 h, and identified by the surgeons and pathologists together. The pathologists then prepared, stained and read the specimen slices. Pathological results were assessed with the 8th Edition of American Joint Committee on Cancer (AJCC) stages [14]. Follow-up examinations were conducted once every 3-4 months during the first 2 years after operation, once every 6 months from 3 to 5 years after operation, and thereafter one time each year. The primary outcome was overall survival (OS). OS was defined as the time from operation to death or follow-up.

\section{Statistical analyses}

Continuous variables were reported with mean and standard deviation. Categorical variables were reported with number and percentage. Univariate and multivariate Logistic regression analyses were applied to analyze whether 
preoperative variables were significant predictors of pathological stages. Univariate and multivariate Cox regression analyses were applied to analyze whether preoperative variables were significant predictors of OS. Two-sided $P$ values $<0.05$ were considered as statistical significant. All analyses were conducted with Statistic Package for Social Science software version 20 (SPSS, Chicago, IL, USA).

\section{Results}

\section{Demographics}

As described in Table 1, patients had a mean age of 59 \pm 9.35 years. Females accounted for $38.5 \%$. Mean levels of SUVmax, CEA and CA19-9 were $5.70 \pm 2.76,3.95 \pm$ $4.16 \mathrm{ng} / \mathrm{mL}$ and $321.62 \pm 780.71 \mathrm{kU} / \mathrm{L}$.

\section{Predictors of AJCC stages}

In univariate Logistic regression analysis (Table 2), preoperative SUVmax, CEA and CA19-9 levels $(p<0.05$ for all) rather than other preoperative variables $(p>0.05$ for all) were significantly related to AJCC stages. Multivariate Logistic regression analysis (Table 2) showed that preoperative SUVmax and CA19-9 levels ( $\mathrm{p}<0.05$ for all) rather than other preoperative variables ( $p>0.05$ for all) were significantly associated with AJCC stages.

Table 1 Description of patients with PDAC after PD

\begin{tabular}{ll}
\hline Variables & Description \\
\hline Age, year & $59 \pm 9.35$ \\
Females (\%) & $42(38.5)$ \\
BMl & $23.25 \pm 3.42$ \\
Operative histories (\%) & $8(7.3)$ \\
Diabetes mellitus (\%) & $18(16.5)$ \\
Hypertension (\%) & $29(26.6)$ \\
Abdominal pain (\%) & $32(29.4)$ \\
Back pain (\%) & $14(12.8)$ \\
Hemoglobin & $129.17 \pm 14.66$ \\
Albumin & $39.00 \pm 3.82$ \\
Total bilirubin & $97.75 \pm 106.47$ \\
CEA & $3.95 \pm 4.16$ \\
CA19-9 & $321.62 \pm 780.71$ \\
SUVmax & $5.70 \pm 2.76$ \\
AJCC & \\
1 & $57(52.3)$ \\
2-4 & $52(47.7)$ \\
OS, month & $21 \pm 14.50$
\end{tabular}

Abbreviations: AJCC: American Joint Committee on Cancer; BMl: body mass index; CA19-9: carbohydrate antigen 19-9; CEA: carcino-embryonic antigen; SUVmax: maximum standardized uptake value; OS: overall survival; PDAC: pancreatic duct adenocarcinoma; PD: pancreaticoduodenectomy

\section{Survival analysis}

Mean OS was $21 \pm 14.50$ months. In univariate Cox regression analysis (Table 3), age, SUVmax, CEA and CA19-9 levels before operation ( $\mathrm{p}<0.05$ for all) rather than other preoperative variables $(\mathrm{p}>0.05$ for all) were significantly related to OS. Multivariate Cox regression analysis (Table 3 ) showed that age, SUVmax and CA199 levels before operation $(p<0.05$ for all) rather than other preoperative variables $(p>0.05$ for all) were significantly associated with OS.

\section{Discussion}

PDAC has a growing trend of incidence and accounts for $1-2 \%$ of malignant cancers $[1,2]$. Although more patients with PDAC have received the PD, they have not obtained significant improved OS [3-5]. Early and individualized operative strategy and perioperative management have the potential to improve the prognosis of patients with PDAC after PD [6, 7]. It results in a greater focus of preoperative variables predicting the OS [8]. The results from the current study confirmed that preoperative SUVmax and CA19-9 levels were independently prognostic predictors of patients with PDAC after PD.

${ }^{18}$ F-FDG PET/CT is a high-tech imaging method not only accurately indicating anatomical image but also effectively displaying functional metabolism [15]. ${ }^{18} \mathrm{~F}-\mathrm{FDG}$ has become extensively applied as a tracer of PET/CT in clinical imaging of PDAC [16]. As PDAC cells progress and anaerobic glycolysis increases, ${ }^{18} \mathrm{~F}$-FDG is taken up more by PDAC cells, converted into $6-\mathrm{P}-{ }^{18} \mathrm{~F}-\mathrm{FDG}$, and stored in PDAC cells. Previous studies have shown that SUVmax plays a significant role in not only the diagnosis, but also staging and prognosis in patients with PDAC [17, 18]. However, conflicting results have been published regarding the prognostic value of SUVmax [19-21]. Moreover, previous studies exploring the value of SUVmax for staging and prognosis have been limited, especially in Chinese patients with PDAC [22]. The current study indicated that SUVmax was a prognostic predictor of OS, suggesting that ${ }^{18} \mathrm{~F}$-FDG-PET/CT might serve as an important imaging method that applied to stratify the patient with PDAC and predict the prognosis of patients after PD.

As one cancer marker often applied to screen the PDAC, CA19-9 is the mucoprotein present in pancreatic, biliary, gastric and intestinal epithelium cells [23]. On the one hand, PDAC cells grow up, invade and injure the normal pancreatic and biliary cells, leading to their release of CA19-9; on the other hand, PDAC cells release the CA19-9 by themselves as PDAC grows up. Previous studies have investigated the potential prognostic value of preoperative CA19-9 levels in patients with PDAC [13] However, preoperative variables that may 
Table 2 Associations between preoperative variables and AJCC stages in univariate and multivariate Logistic analyses

\begin{tabular}{|c|c|c|c|c|c|c|}
\hline Variables & $\mathrm{OR}^{\mathrm{a}}$ & $95 \mathrm{Cl}^{\mathrm{a}}$ & $P$ value $^{a}$ & $O R^{b}$ & $95 \mathrm{Cl}^{\mathrm{b}}$ & $P$ value $^{b}$ \\
\hline Age & 0.996 & $0.957-1.037$ & 0.844 & 0.998 & $0.941-1.058$ & 0.936 \\
\hline Females & 0.994 & $0.459-2.152$ & 0.988 & 0.977 & $0.318-3.055$ & 0.968 \\
\hline BMI & 1.057 & $0.945-1.183$ & 0.329 & 1.093 & $0.937-1.275$ & 0.257 \\
\hline Operative histories & 0.340 & $0.065-1.765$ & 0.199 & 0.259 & $0.032-2.071$ & 0.203 \\
\hline Diabetes mellitus & 0.489 & $0.169-1.416$ & 0.187 & 1.033 & $0.275-3.879$ & 0.962 \\
\hline Hypertension & 0.706 & $0.299-1.667$ & 0.427 & 0.593 & $0.184-1.914$ & 0.382 \\
\hline Abdominal pain & 1.949 & $0.843-4.503$ & 0.118 & 1.390 & $0.472-4.091$ & 0.550 \\
\hline Back pain & 1.545 & $0.498-4.797$ & 0.451 & 2.974 & $0.670-13.198$ & 0.152 \\
\hline Hemoglobin & 1.002 & $0.976-1.028$ & 0.902 & 1.013 & $0.966-1.063$ & 0.586 \\
\hline Albumin & 0.975 & $0.883-1.076$ & 0.616 & 0.914 & $0.780-1.071$ & 0.266 \\
\hline Total bilirubin & 1.001 & $0.998-1.005$ & 0.532 & 0.999 & $0.994-1.005$ & 0.798 \\
\hline CEA & 1.259 & $1.056-1.501$ & 0.010 & 1.187 & $0.962-1.465$ & 0.109 \\
\hline CA19-9 & 1.003 & $1.001-1.005$ & 0.001 & 1.003 & $1.001-1.005$ & 0.009 \\
\hline SUVmax & 1.277 & $1.080-1.510$ & 0.004 & 1.322 & $1.073-1.629$ & 0.009 \\
\hline
\end{tabular}

Abbreviations: BMI: body mass index; CA19-9: carbohydrate antigen 19-9; CEA: carcino-embryonic antigen; OR: odds ratio; SUVmax: maximum standardized uptake value

Notes: ${ }^{a}$ univariate Logistic regression analysis; ${ }^{b}$ multivariate Logistic regression analysis

play a predictive role in patients with PDAC are poorly understood in previous studies, especially in Chinese patients. The current study proved that preoperative CA19-9 levels were associated with significant reduced OS and more poor differentiation following PDAC. These findings supported that preoperative CA19-9 levels were not only indicative of tumour burden, but also that preoperative CA19-9 levels might act as a marker of biological aggressiveness [24].

\section{Conclusions}

The current study demonstrated that preoperative SUVmax and CA19-9 levels independently predicted pathological stages and OS of patients with PDAC after PD. These preoperative variables might have significant prognostic implication in patients with PDAC after PD. Patients with abnormal SUVmax and CA19-9 levels should be paid special attention to in operative strategy and perioperative management.

Table 3 Associations between preoperative variables and OS in univariate and multivariate Cox regression analyses

\begin{tabular}{|c|c|c|c|c|c|c|}
\hline Variables & $O R^{a}$ & $95 \mathrm{Cl}^{\mathrm{a}}$ & $P$ value $^{a}$ & $O R^{b}$ & $95 \mathrm{Cl}^{\mathrm{b}}$ & $P$ value $^{b}$ \\
\hline Age & 1.027 & $1.002-1.053$ & 0.035 & 1.034 & $1.004-1.066$ & 0.028 \\
\hline Females & 1.111 & $0.711-1.738$ & 0.643 & 0.911 & $0.544-1.524$ & 0.722 \\
\hline BMI & 0.968 & $0.909-1.032$ & 0.324 & 0.971 & $0.903-1.044$ & 0.429 \\
\hline Operative histories & 1.455 & $0.630-3.358$ & 0.380 & 1.586 & $0.633-3.976$ & 0.325 \\
\hline Diabetes mellitus & 0.669 & $0.353-1.267$ & 0.217 & 0.581 & $0.284-1.189$ & 0.137 \\
\hline Hypertension & 1.158 & $0.716-1.871$ & 0.550 & 1.120 & $0.632-1.986$ & 0.697 \\
\hline Abdominal pain & 1.265 & $0.794-2.017$ & 0.323 & 1.172 & $0.695-1.977$ & 0.552 \\
\hline Back pain & 0.960 & $0.506-1.823$ & 0.901 & 1.355 & $0.658-2.791$ & 0.410 \\
\hline Hemoglobin & 0.990 & $0.976-1.004$ & 0.167 & 0.995 & $0.973-1.017$ & 0.636 \\
\hline Albumin & 0.983 & $0.929-1.039$ & 0.545 & 1.005 & $0.929-1.088$ & 0.899 \\
\hline Total bilirubin & 1.000 & $0.998-1.002$ & 0.888 & 1.000 & $0.998-1.003$ & 0.794 \\
\hline CEA & 1.104 & $1.048-1.163$ & $<0.001$ & 1.050 & $0.966-1.141$ & 0.250 \\
\hline CA19-9 & 1.001 & $1.000-1.001$ & $<0.001$ & 1.000 & $1.000-1.001$ & 0.035 \\
\hline SUVmax & 1.129 & $1.052-1.213$ & 0.001 & 1.136 & $1.051-1.228$ & 0.001 \\
\hline
\end{tabular}

Abbreviations: BMI: body mass index; CA19-9: carbohydrate antigen 19-9; CEA: carcino-embryonic antigen; OR: odds ratio; OS: overall survival; SUVmax: maximum standardized uptake value 


\begin{abstract}
Abbreviations
${ }^{18}$ F-FDG: ${ }^{18}$ F-fluorodeoxyglucose; CA19-9: Carbohydrate antigen 19-9; CEA: Carcino-embryonic antigen; PD: Pancreaticoduodenectomy; PDAC: Pancreatic duct adenocarcinoma; PET/CT: Positron emission tomography/computed tomography; SUVmax: Maximum standardized uptake value
\end{abstract}

\section{Acknowledgments}

We are grateful to all study participants for their participation in the study.

\section{Funding}

The authors have no funding to be reported.

\section{Availability of data and materials}

In attempt to preserve the privacy of patients, clinical data of patients will not be shared; data can be available from authors upon request.

\section{Authors' contributions}

Conceived and designed the experiments: $X G, R Z, C L, R L, Z Z, Y G, Y X$; Performed the experiments: $X G, R Z, C L, R L, Z Z, Y G, Y X$; Analyzed the data: $X G, R Z, C L, R L, Z Z, Y G, Y X$; Contributed reagents/materials/analysis tools: $X G$, $R Z, C L, R L, Z Z, Y G, Y X$; Wrote the paper: $X G, R Z, C L, R L, Z Z, Y G, Y X$. All authors read and approved the final version of the manuscript.

\section{Ethics approval and consent to participate}

The study protocol was approved by Ethics Committee of Chinese People's Liberation Army General Hospital (Beijing, China). Each participant provided written informed consent to be included in the study.

\section{Consent for publication}

Not applicable.

\section{Competing interests}

The authors declare that they have no competing interests.

\section{Publisher's Note}

Springer Nature remains neutral with regard to jurisdictional claims in published maps and institutional affiliations.

\section{Received: 17 November 2018 Accepted: 8 May 2019}

\section{Published online: 15 May 2019}

\section{References}

1. Siegel RL, Miller KD, Jemal A. Cancer statistics, 2016. CA Cancer J Clin. 2016; 66:7-30.

2. Okano K, Suzuki Y. Strategies for early detection of resectable pancreatic cancer. World J Gastroenterol. 2014:20:11230-40.

3. Schnelldorfer T, Ware AL, Sarr MG, et al. Longterm survival after pancreatoduodenectomy for pancreatic adenocarcinoma: is cure possible? Ann Surg. 2008;247:456-62.

4. Adham M, Jaeck D, Le Borgne J, et al. Longterm survival (5-20 years) after pancreatectomy for pancreatic ductal adenocarcinoma: a series of 30 patients collected from three institutions. Pancreas. 2008;37:352-7.

5. Katz $\mathrm{MH}$, Wang $\mathrm{H}$, Fleming JB, et al. Longterm survival after multidisciplinary management of resected pancreatic adenocarcinoma. Ann Surg Oncol. 2009;16:836-47.

6. Jamieson NB, Foulis AK, Oien KA, et al. Positive mobilization margins alone do not influence survival following pancreaticoduodenectomy for pancreatic ductal adenocarcinoma. Ann Surg. 2010;251:1003-10.

7. Cleary SP, Gryfe R, Guindi M, et al. Prognostic factors in resected pancreatic adenocarcinoma: analysis of actual 5-year survivors. J Am Coll Surg. 2004; 198:722-31.

8. Z Zhu D, Wang L, Zhang H, et al. Prognostic value of ${ }^{18} \mathrm{~F}-\mathrm{FDG}-\mathrm{PET} / \mathrm{CT}$ parameters in patients with pancreatic carcinoma: a systematic review and meta-analysis. Medicine (Baltimore). 2017;96(33):e7813.

9. Vriens D, Visser EP, de Geus-Oei LF, et al. Methodological considerations in quantification of oncological FDG PET studies. Eur J Nucl Med Mol Imaging. 2010;37:1408-25.

10. Dholakia AS, Chaudhry M, Leal JP, et al. Baseline metabolic tumor volume and total lesion glycolysis are associated with survival outcomes in patients with locally advanced pancreatic cancer receiving stereotactic body radiation therapy. Int J Radiat Oncol Biol Phys. 2014;89:539-46.

11. Smith RA, Bosonnet L, Ghaneh P, et al. Preoperative CA19-9 levels and lymph node ratio are independent predictors of survival in patients with resected pancreatic ductal adenocarcinoma. Dig Surg. 2008;25(3):226-32.

12. Safi F, Schlosser W, Falkenreck S, et al. Prognostic value of CA19-9 serum course in pancreatic cancer. Hepatogastroenterology. 1998;45:253-9.

13. Ferrone CR, Finkelstein DM, Thayer SP, et al. Perioperative CA19-9 levels can predict stage and survival in patients with pancreatic ductal adenocarcinoma. J Clin Oncol. 2006;24:2897-902.

14. Chun YS, Pawlik TM, Vauthey JN. 8th edition of the AJCC Cancer staging manual: pancreas and hepatobiliary cancers. Ann Surg Oncol. 2018;25(4): 845-7.

15. Ell PJ. PET/CT in oncology: a major technology for cancer. Chang Gung Med J. 2005:28:274-83.

16. von Schulthess GK, Steinert HC, Hany TF. Integrated PET/CT: current applications and future directions. Radiology. 2006;238:405-22.

17. Grassetto G, Rubello D. Role of FDG-PET/CT in diagnosis, staging, response to treatment, and prognosis of pancreatic cancer. Am J Clin Oncol. 2011;34: $111-4$.

18. De Gaetano AM, Rufini V, Castaldi P, et al. Clinical applications of (18) F-FDG PET in the management of hepatobiliary and pancreatic tumors. Abdom Imaging. 2012;37:983-1003.

19. Im HJ, Oo S. JungW, et al. prognostic value of metabolic and volumetric parameters of preoperative FDG-PET/CT in patients with resectable pancreatic cancer. Medicine. 2016;95:e3686.

20. Lee JW, Kang CM, Choi HJ, et al. Prognostic value of metabolic tumor volume and total lesion glycolysis on preoperative (1)(8) F-FDG PET/CT in patients with pancreatic cancer. J Nucl Med. 2014;55:898-904.

21. Xu HX, Chen T, Wang WQ, et al. Metabolic tumour burden assessed by ${ }^{18} \mathrm{~F}-$ FDG PET/CT associated with serum CA19-9 predicts pancreatic cancer outcome after resection. Eur J Nucl Med Mol Imaging. 2014;41:1093-102.

22. Wang Z, Chen JQ, Liu JL, et al. FDG-PET in diagnosis, staging and prognosis of pancreatic carcinoma: a meta-analysis. World J Gastroenterol. 2013;19: 4808-17.

23. Ballehaninna UK, Chamberlain RS. The clinical utility of serum CA 19-9 in the diagnosis, prognosis and management of pancreaticadenocarcinoma: an evidence based appraisal. J Gastrointest Oncol. 2012;3(2):105-19.

24. Bogoevski D, Yekebas EF, Schurr P, et al. Mode of spread in the early phase of lymphatic metasis in pancreatic ductal adenocarcinoma: prognostic significance of nodal microinvolvement. Ann Surg. 2004;240:993-1000.

\section{Ready to submit your research? Choose BMC and benefit from:}

- fast, convenient online submission

- thorough peer review by experienced researchers in your field

- rapid publication on acceptance

- support for research data, including large and complex data types

- gold Open Access which fosters wider collaboration and increased citations

- maximum visibility for your research: over $100 \mathrm{M}$ website views per year

At BMC, research is always in progress.

Learn more biomedcentral.com/submissions 